\title{
Simulation of physical habitat in Ayuquila-Armeria river in the west of Mexico
}

\author{
Demetrio Meza Rodríguez ${ }^{a}$ - Luis Manuel Martínez Riveraa - José Luis Olguín \\ López $^{a}$ - Ángel Aguirre Garcia ${ }^{a}$
}

\begin{abstract}
Resumen: en México, en 2012, se publicó una norma para la determinación del caudal ecológico en los ríos y arroyos que han sido severamente degradados por el desvió de agua de estos para actividades productivas, principalmente agropecuarias e industriales. Esta norma considera la simulación del hábitat físico como una medida para la determinación del caudal dentro del Instream Flow Incremental Methodology (IFIM). En este estudio, fueron generadas las simulaciones de hábitat físico, combinadas con las curvas de idoneidad de peces. Para generar las simulaciones de hábitat, se seleccionó un tramo de río de 706 metros, donde se midieron los parámetros de profundidad, velocidad y tipo de sustrato. Se usó el software iRIC 1.5 para calibrar y simular los caudales con el hábitat físico, donde la principal entrada de información fue un levantamiento topográfico del río y la construcción de las curvas de idoneidad de las especies de peces seleccionadas. Como resultado se obtuvieron simulaciones a diferente caudal y diferente hábitat potencial útil (HPU) para cada una de las especies de interés. Los resultados mostraron que cuando cae el caudal a caudales inferiores a $1\left(\mathrm{~m}^{3} \mathrm{~s}^{-1}\right)$ y superiores a los $200\left(\mathrm{~m}^{3} \mathrm{~s}^{-1}\right)$, hay decaimiento del HPU, muy pobre para las cuatro especies. Pocos estudios en Latinoamérica se han desarrollado para este tipo de análisis, por lo que esta investigación contribuye a proceso metodológico que ayudará a mejorar la investigación de caudal ambiental en ríos.
\end{abstract}

Palabras clave: modelo bidimensional; modelo hidrodinámico; especie indicadora; régimen hidrológico.

Fecha recepción: 30 de octubre de 2017 Fecha aprobación: 22 de abril de 2019

Cómo citar: D. Meza Rodríguez, L. M. Martínez Rivera, J. L. Olguín López, Á. Aguirre García, “Simulation of physical habitat in Ayuquila-Armeria river in the west of Mexico, Ciencia e Ingenieria Neogranadina, vol. 29, no. 2, pp. 53-68, 2019. Doi: https://doi.org/10.18359/rcin.3128

a Universidad de Guadalajara, México.

Correo electrónico: demetrio.meza@cucsur.udg.mx. ORCID: https://orcid.org/0000-0002-5855-6939

b Universidad de Guadalajara, México.

Correo electrónico:: Imartinez@cucsur.udg.mx. ORCID: https://orcid.org/0000-0002-7050-9385

c Universidad de Guadalajara, México.

Correo electrónico: olguin@cucsur.udg.mx.

d Universidad de Guadalajara, México.

Correo electrónico: aguirrea@cucsur.udg.mx. ORCID: https://orcid.org/0000-0001-5944-6750 
Abstract: In Mexico, until 2012 was published a norm for the determination of the ecological flow in rivers and streams, which have been severely degraded by water diversion for agricultural and industrial activities. This standard considers the simulation of the physical habitat as a measure for the determination of the flow within the Instream Flow Incremental Methodology (IFIM). In this study, simulations of physical habitat were generated, combined with fish fitness curves. To generate the habitat simulations, a section of river with 706 meters was selected, where the parameters of depth, speed and type of substrate were measured. The iRIC 1.5 software was used to calibrate and simulate the flows with the physical habitat, where the main information input was a topographic survey of the river, and the construction of the suitability curves for the selected fish species. As a result, simulations were obtained at different flow rates and different Weighted Usable Area (WUA) for each of the species of interest. The results showed us that when the flow falls at lower flow rates lower than 1 $\left(\mathrm{m}^{3} \mathrm{~s}^{-1}\right)$ and higher than $200\left(\mathrm{~m}^{3} \mathrm{~s}^{-1}\right)$, it shows a very poor WUA decay for the four species. Few studies in Latin America have been developed for this type of analysis, so this study contributes significantly as a methodological process that will help improve the research of environmental flow in rivers.

Keywords: two-dimensional model; hydrodynamic model; indicator species; hydrological regime. 


\section{Introducción}

La simulación de hábitat físico es una herramienta numérica que permite cuantificar dicho hábitat en términos de la profundidad del agua $(\mathrm{m})$, la velocidad $\left(\mathrm{m} \mathrm{s}^{-1}\right)$ y el tipo de sustrato $(\mathrm{m})$, en una descarga específica para un caudal dado [1]. Además, predice el impacto de un cambio en el caudal en la disponibilidad de hábitat para las especies seleccionadas como objetivo [2]-[5]. Estas simulaciones de hábitat físico son un requisito esencial para evaluar la salud [6]-[8], el manejo, el inventario y la restauración de los ríos [8]-[11].

Los modelos de simulación de hábitat físico se desarrollan en la interfaz de dos disciplinas históricamente diferentes: la ecología y la hidráulica, combinando los procesos físicos y su influencia en la ecología acuática y la biología [12]. La necesidad de resolver problemas en el manejo del agua ha impulsado su desarrollo como un campo distinto de investigación aplicada con un interés creciente en todo el mundo [12]-[15]. La simulación de hábitat físico tiene como objetivo lograr una evaluación exhaustiva; los científicos han desarrollado centenares de métodos y técnicas de evaluación del caudal del rio, para diversas configuraciones y con diferentes bases teóricas [3], [16].

Entre los modelos más usados está el unidimensional de simulación hidráulica y de hábitat acuático, Physical Habitat Simulation System (РнAвsim) [17]. Este ha sido el más usado en estudios de simulación de hábitat físico en el mundo, en especial en Norte América [18]-[20]. Junto a este, existe el modelo en dos dimensiones, River2D [21], que se usa para los estudios de simulación de hábitat acuático [22]-[25].

PHABsim y River2D son empleados en la Instream Flow Incremental Methodology (IFIM) [26], y quizás son los procedimientos más conocidos y documentados para la planificación de los recursos hídricos [3]. Estos modelos simulan el hábitat idóneo en las corrientes de agua, y los resultados se muestran en valores de hábitat potencial útil (HPU), que representa el área utilizable de hábitat idóneo para las especies acuáticas en los tramos de interés [27].
En México, en 2012, se publicó la Norma Mexicana NMX-AA-159-SCFI-2012, que establece el procedimiento para la determinación del caudal ecológico en cuencas hidrológicas [28]. La norma considera la simulación de hábitat fisico para definir el caudal mínimo ecológico, a partir de un estudio de todos los factores y condicionantes del hábitat para una o varias especies objetivo del ecosistema fluvial [29]. Sin embargo, la relación entre los conocimientos de hábitat físico (profundidad, anchura del cauce, pendiente de las orillas, velocidad del agua, tipo de sustrato, vegetación acuática y sombreado), los biotopos (el cauce, la ribera y la llanura de inundación), los parámetros físicos-químicos (temperatura, $\mathrm{pH}$, conductividad, sólidos disueltos y oxígeno disuelto) y los ecológicos (alimentación, reproducción, competencia, crecimiento, entre otros), o la caracterización de los hábitats funcionales en México y Latinoamérica, presentan un desarrollo incipiente.

El río Ayuquila-Armería es uno de los ríos más importantes en el occidente mexicano; el agua que fluye en él es almacenada en cuatro grandes embalses: Tacotán, General Ramón Corona Madrigal (Trigomil), Basilio Badillo (Las Piedras) y El Nogal. Esta retención sirve para el aprovechamiento de los distritos de riego 094 en el Estado de Jalisco y 053 en el Estado de Colima, donde, durante la temporada de lluvias, se minimiza el flujo natural y se almacena para el riego del siguiente año [30]. El desvío y la retención han afectado la biota del río [29], [31] y [32].

Considerando las obligaciones normativas mencionadas y la gran importancia del río AyuquilaArmería, el objetivo de este estudio es generar las simulaciones de hábitat físico, utilizando las curvas de idoneidad de hábitat de las especies: Nandopsis istlanum (Jordan y Schneider, 1899), Astyanax cf. aeneus (Günther, 1860), Poecilia butleri (Jordan, 1889) y Ilyodon furcidens (Jordan y Gilbert, 1882), que se encuentran dentro del río Ayuquila-Armería. La combinación de la hidráulica del río y las curvas de idoneidad determinarán el HPU para cada caudal simulado, además de relacionar las simulaciones con las series de tiempo del régimen hidrológico actual, utilizando el software International River Interface Cooperative 
(iRIC 1.5) en su plataforma FaSTMECH, y siguiendo la metodología IFIM. Los resultados conseguidos se utilizan en la estimación del caudal mínimo ecológico dentro del río para las especies objetivo seleccionadas. La información lograda proporcionará datos confiables a los gestores en la toma de decisiones, para un mejor uso y manejo de los recursos hídricos del río.

\section{Materiales y métodos}

\section{Tramo de estudio}

El tramo para la simulación de hábitat físico se ubicó a $30 \mathrm{~km}$ aguas abajo de la confluencia de los ríos Ayuquila y Tuxcacuesco: en las coordenadas $19^{\circ} 28^{\prime} 56,68^{\prime \prime}(\mathrm{N})$ y $103^{\circ} 50^{\prime} 08,64^{\prime \prime}$ (O) (Figura 1), y a una distancia aguas abajo de las presas: Trigomil y Las Piedras con 110 y $85 \mathrm{~km}$, respectivamente. La distancia de las presas y la dinámica hidrológica que presenta en el tramo seleccionado se asocian a un régimen natural [26]. El tramo seleccionado limita entre los estados de Jalisco y Colima en el occidente de México, la cuenca vertiente drena una superficie aproximada de $9,864 \mathrm{~km}^{2}$ con un trayecto de $321 \mathrm{~km}$ desde su nacimiento hasta su desembocadura [29]. El caudal es extremadamente variable, con $10 \mathrm{~m}^{3} \mathrm{~s}^{-1}$ en la época seca del año y valores por arriba de los $160 \mathrm{~m}^{3} \mathrm{~s}^{-1}$ en la época de lluvias, con un flujo permanente durante todo el año. El clima es semiárido, con una precipitación promedio anual de $836 \mathrm{~mL}$ y una temperatura promedio de $21^{\circ} \mathrm{C}[33]$.

\section{Selección del tramo de estudio}

El tramo seleccionado tiene una longitud de 706 metros, incluyendo las diferentes unidades morfodinámicas (pozas, rápidos, rápidos someros, remansos, tablas e isletas) características del río. Con el manejo del equipo topográfico (estación total Sokkia 630 R y GPS Astech Promark 3), se levantaron 62 secciones de río, con una distancia aproximada entre secciones de 10 metros (Figura 1), con fecha de referencia del 12 al 14 de junio 2013. Cada sección consistió en un transecto perpendicular a la dirección de flujo, partiendo del punto de mayor inundación de un lado del río hasta el otro [29].
En cada una de las secciones del río se instauraron puntos de medición de los parámetros: profundidad de la columna de agua (m), velocidad $\left(\mathrm{m} \mathrm{s}^{-1}\right)$ y granulometría del sustrato $(\mathrm{mm})$; este último utiliza la técnica Pebble Count en transectos [34]. Estas mediciones se establecieron considerando los cambios trasversales de la pendiente, la profundidad, la velocidad y el tipo de sustratos, cada punto quedó en coordenadas UTM WGS84 13 Norte.

Para estimar el caudal circulante dentro del sitio de estudio, en cada punto donde se estimó la altura de la lámina de agua, a diferentes distancias en la sección transversal, se definieron unas celdas donde se midió la velocidad del agua, a una profundidad de la superficie de 0,6 veces del calado [29]. Para el correcto ajuste del modelo hidráulico, se realizaron dos mediciones del caudal circulante: una sección aguas arriba del sitio de trabajo y otra en la salida la sección aguas abajo del sitio del tramo en estudio) [29]. Se usó el método de velocidad-área para estimar el caudal circulante [35].

\section{Simulación hidráulica}

El software iRIC 1.5 implementa la dinámica general de fluidos, y fue desarrollado originalmente por la National Aeronautics and Space Administration (NASA), para el modelado estable y rápido en 2D, más la dinámica de fluidos complejos [23] y [36]. Este software hidrodinámico, en su plataforma conocida como FaSTMECH (Flow and Sediment Transport with Morphological Evolution of Channels), utiliza el modelado del caudal en dos dimensiones (2D), sus tiempos de ejecución son rápidos y su uso es fácil; las ecuaciones que utiliza se emiten en un sistema de coordenadas curvilíneas ortogonales, que se ajustan al canal del río y se describen solo por un trayecto de línea central, ancho y con resolución espacial; la ruta se elige en una línea recta y la cuadrícula o malla es cartesiana [37] y [38].

\section{Parámetros de entrada y condiciones límite}

Como dato de partida, iRIC 1.5 usa la representación del Modelo Digital del Terreno (MDT), que se obtiene de la topografía detallada del sitio de estudio. El MDT es almacenado en un formato de red irregular triangulada (TIN); a este se incorporaron los datos 
topográficos y batimétricos en el MDT, para complementar los datos originales y verificar la "superficie" resultante (topográfica y batimétrica) [38] y [39].

La plataforma FaSTMECH dentro de iRIC 1.5 requiere definir tres condiciones para obtener una solución válida y estable. Estos valores dependen de las características hidráulicas del cauce, que son representadas por el dominio del modelo. Los valores óptimos se resuelven por lo general a través de un proceso de prueba y error [40].

La primera condición fue la estimación del caudal circulante en $\mathrm{m}^{3} \mathrm{~s}^{-1}$ aguas arriba, donde inicia el tramo de estudio, y se obtuvo de los datos observados en las campañas de campo. La segunda condición fue la elevación de la superficie de la lámina de agua a la salida del tramo de estudio (aguas abajo), que se consiguió del levantamiento topográfico del río a una elevación en metros sobre el nivel del mar.

La tercera condición de entrada corresponde al valor $n$ Manning, que generalmente varía entre 0,030 a 0,038 [41]. En este estudio la rugosidad del canal fue representada por coeficientes de arrastre adimensionales y se calculó con base en el material de lecho, como el tamaño medio del sustrato encontrado en el tramo de estudio; por tanto, no se ajustó durante la calibración [38] y [39].

\section{Calibración y validación}

Con la información del levantamiento topográfico, fueron definidos 62 puntos de elevación de la superficie de la lámina de agua, identificados como el Thalweg a lo largo del tramo de estudio (Figura 1). Con la información del caudal circulante observado y asociado a la elevación de la superficie de la lámina de agua del Thalweg es posible comparar el modelo predicho [42] y los parámetros del modelo: caudal circulante observado $\left(\mathrm{m}^{3} \mathrm{~s}^{-1}\right)$, elevación de la superficie de la lámina de agua $(\mathrm{m})$ encontrada a la salida del tramo de estudio y la rugosidad constante (m). Dentro de FaSTMECH en el módulo Relaxation Parameters, para calibrar el modelo se ajustó la respuesta de la elevación de la superficie de la lámina de agua observada $v s$. la calculada por el programa, y que sean coincidentes para los 62 puntos del Thalweg, hasta obtener una solución satisfactoria.

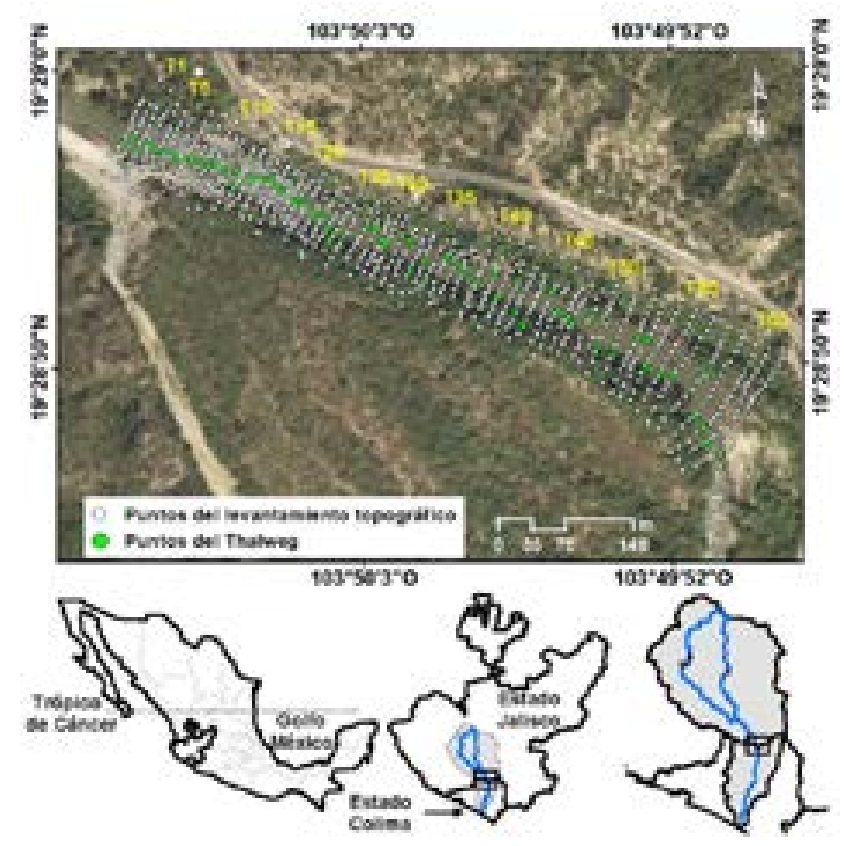

Fig. 1. Localización del tramo de estudio,

Fuente: elaboración propia.

Nota: en el tramo se localizan los puntos del levantamiento topográfico y el Thalweg (zona más profunda del río donde la corriente es más fuerte), se ubican dentro de la cuenca del río Ayuquila-Armería, se distribuye entre los estados de Jalisco y Colima en el occidente de México. T=transecto. 
Para validar el modelo de simulación, se usó el módulo Verification dentro de la plataforma FaSTMECH, y se obtuvo el error cuadrático medio (ЕСM) de la elevación de la superficie de la lámina de agua $(\mathrm{m})$ calculada por el programa $v s$. observada en las campañas de campo. Al mismo tiempo, se empleó el coeficiente de determinación $\left(\mathrm{R}^{2}\right)$ para medir la asociación del modelo calculado con el observado. Con los valores resultantes del ECM y $\mathrm{R}^{2}$, de acuerdo con la calidad de asociación, el modelo será validado. Después de aprobarse el modelo hidráulico, se generan las diferentes simulaciones a diferente caudal a lo largo del tramo de estudio, para las especies seleccionadas y para un horizonte de tiempo de 31 años (1971-2001) del régimen hidráulico actual.

\section{Selección de las curvas de idoneidad}

Una vez medidas las características hidráulicas del cauce y calibrado el modelo de simulación hidráulico, fue necesario seleccionar las especies indicadoras del tramo de estudio. Para esto se llevó a cabo un análisis de las bases de datos del programa de monitoreo bimensual de largo plazo (1998-2011), de calidad de agua y peces dentro del río Ayuquila-Armería [33], del proyecto regional Manejo y conservación de los recursos naturales del río Ayuquila-Armería, Departamento de Ecología y Recursos Naturales de la Universidad de Guadalajara (comunicación personal, Luis Manuel Martínez). Las especies seleccionadas como indicadoras del tramo de estudio fueron: N. istlanum, A. cf. aeneus, P. butleri y I. furcidens.

Las curvas de idoneidad se consiguieron a través de estadísticas con la técnica de histograma de frecuencia para cada variable de interés y para cada especie; por tanto, los datos de cada variable se clasificaron en grupos de intervalos siguiendo los pasos establecidos por [26] y [43] (Figura 2).
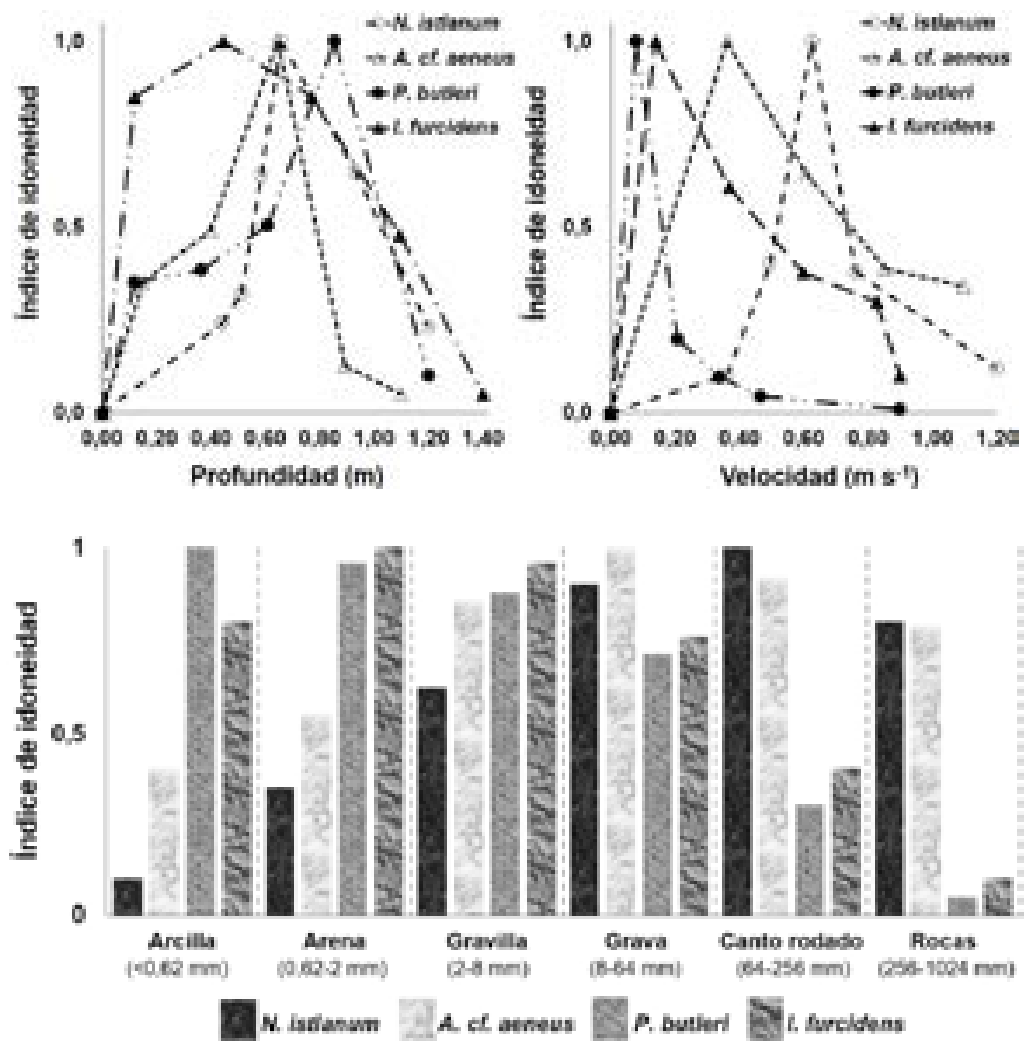

Fig. 2. Curvas de idoneidad de hábitat acuático, para las variables de profundidad, velocidad y tipo de sustratos Fuente: datos del proyecto Manejo y Conservación de los Recursos Naturales del río Ayuquila-Armería de 1998-2011. Nota: el último se muestra con forma de barras para las especies seleccionadas como indicadoras del tramo de río. 
Los tres factores escogidos, velocidad, profundidad y tipo de sustratos, fueron integrados para relacionar las características físicas del tramo fluvial seleccionado, con las necesidades de hábitat de las especies que se eligieron.

\section{Simulación del hábitat}

Para simular el hábitat físico se empleó la aplicación Habitat Builder, que se encuentra dentro de la plataforma FaSTMECH, y se usó la información de las curvas de idoneidad mencionadas anteriormente.

El módulo Habitat Builder permite el cálculo de las estimaciones de área utilizables para las variables independientes: profundidad, velocidad y tipo de sustratos. Para cada variable se usó el método de interpolación Tin (red de triángulos irregulares). La idoneidad final de esta celda se obtiene promediando (p.e. media geométrica) la idoneidad interpolada de las curvas de idoneidad [38]. Habitat Builder define una función de hábitat compuesta por uno o más predictores (curvas de idoneidad) para mapear el valor de la función de hábitat a la celda del modelo, y calcula un HPU que consiste en la suma para cada caudal evaluado del área o pixel de simulación hidráulica ponderado por la idoneidad conjunta [38].

\section{Criterios de idoneidad del hábitat}

Para producir una medida representativa del hábitat físico disponible en función de la descarga (caudal), los parámetros (profundidad, velocidad y tipo de sustratos) fueron combinados en la plataforma FaSTMECH, con la preferencia de las especies. Esta combinación genera el Índice de Aptitud Combinada (IAC) y deduce que los parámetros hidráulicos, mencionados anteriormente, son variables independientes que caracterizan el hábitat, los cuales se usan comúnmente para describir la calidad del hábitat a escala del tramo de río seleccionado, y tienen la expresión de la ecuación (1) [40], [44] y [45]:

$$
I A C_{i}=\left(\Pi_{j} I I_{i, j}\right)^{I / m}
$$

Donde $\Pi$ es el operador del producto, los subíndices $i$ y $j$ indican el $i$-th celda y el $j$-th el parámetro físico. El valor de $I A C$ varía espacialmente con la descarga.

El Hábitat Potencial Útil (HPU) y el Hábitat Potencial Hidráulico (HPH), se deriva del IAC y comúnmente utilizado para describir la cantidad del hábitat [46-47]. Tanto HPU como HPH son funciones de descarga y utilizan las siguientes expresiones (2), (3) respectivamente:

$$
\begin{gathered}
H P U=\sum_{I=1}^{\rho} I A C_{i} A_{I} \\
H P H=\frac{H P U}{A_{w}}
\end{gathered}
$$

Donde $p$ es el número de celdas dentro del área mojada $\mathrm{A}_{\mathrm{w}}$ del tramo del río y $\mathrm{A}_{\mathrm{i}}$ es el área de la celda $i$-th. El HPU se expresa en unidades de área de microhábitat por distancia unitaria a lo largo del río (p.e., $\mathrm{m}^{2}$ por $1000 \mathrm{~m}$ ) [40], [48].

\section{Caudal simulado/hábitat potencial útil}

Después de calibrarse el modelo hidráulico, se llevaron a cabo las simulaciones de caudal a: 0,2. 0,3. $0,4.0,5.0,8$ y $1,2,3,4$ hasta alcanzar los $20 \mathrm{~m}^{3} \mathrm{~s}^{-1}$. Para las simulaciones de caudal se obtuvo el HPU para cada una de las especies seleccionadas.

\section{Simulaciones del régimen hidráulico actual}

Para generar el modelamiento hidráulico de caudales se introdujo una serie de caudales observados y se modeló de forma dinámica un horizonte de tiempo para 31 años (1971-2001); esto se hizo a través de la información de la estación hidrométrica 16032 Coliman, localizada en las coordenadas geográficas $18^{\circ} 56^{\prime} 25,26^{\prime \prime} \mathrm{N}$ y $103^{\circ} 56^{\prime} 39,72^{\prime \prime} \mathrm{O}$. Los valores promedio a nivel mensuales de la serie de caudales estudiados fueron simulados para obtener el HPU para cada una de las especies seleccionadas. Los datos fueron extraídos del Banco Nacional de Aguas Superficiales (Bandas) de la Comisión Nacional de Agua (Conagua) [49]. 


\section{Resultados y discusión}

El resultado del levantamiento topográfico en el río fue de 1,055 puntos distribuido en los 706 metros del tramo de estudio (Figura 1), mientras que el del caudal circulante observado para el tramo de estudio fue de 11,50 $\left(\mathrm{m}^{3} \mathrm{~s}^{-1}\right)$, con una elevación de la superficie de la lámina de agua (ESLA) de 618,65 m s. n. m. a la salida del tramo de estudio (aguas abajo). Las características encontradas del lecho del río fueron las siguientes: madera en descomposición $1 \%$, limos y arcillas $1 \%$, arenas $16 \%$, gravas $12 \%$, cantos rodados $11 \%$ y rocas $59 \%$.

La clasificación del tamaño del sustrato resultante fue D16 0,98 mm, D35 120 mm, D50 $680 \mathrm{~mm}$, D65 1200 mm, D84 1600 mm, D95 1900 mm, con un promedio de $39,6 \mathrm{~mm}$. La rugosidad del canal, representada por el coeficiente de arrastre adimensionales, fue estimada en $0,0396 \mathrm{~m}$, con un sesgo (skewness) de -0,79. Este valor negativo indica que los valores estarán más reunidos en niveles superiores a la media aritmética para el tramo de estudio. El tamaño del sustrato fue un aspecto importante para la simulación de hábitat, ya que definió el hábitat físico para los peces. En el tramo las partículas $>1000 \mathrm{~mm}$ se presentaron en los transectos T1 a T20 con pendientes promedio de $1,17 \%$, en los transectos T21 a T40 con pendientes de $0,05 \%$ dominados por limos, arcillas y arenas, y los transectos T41 a T60 con pendientes $0,01 \%$ con presencia de arenas, gravas y gravillas (Figura 1).

\section{Calibración}

El resultado de la relación entre esLa (puntos del Thalweg) y el caudal circulante presentó un buen ajuste entre los valores calculados por el modelo y los observados en las campañas de campo (Figura 3a), con un coeficiente de determinación de 0,9992 (Tabla 2). En el análisis de asociación el ECM entre ESLA calculada y observada fue de 0,040 metros (Tabla 2), lo que muestra un error de EsLA calculada cercano a cero metros (Figura 3b).

Los resultados de evaluación de las diferentes simulaciones mostraron que al calibrar el modelo a $11,50\left(\mathrm{~m}^{3} \mathrm{~s}^{-1}\right)$, las simulaciones generadas a diferente caudal (altos y bajos) expusieron un buen ajuste de acuerdo con $\mathrm{R}^{2}$, con valores por arriba de 0,90 en la mayor parte de las simulaciones (Tabla 1). Las menores asociaciones encontradas fueron para los caudales inferiores a $1\left(\mathrm{~m}^{3} \mathrm{~s}^{-1}\right)$ y los superiores a $200\left(\mathrm{~m}^{3} \mathrm{~s}^{-1}\right)$.
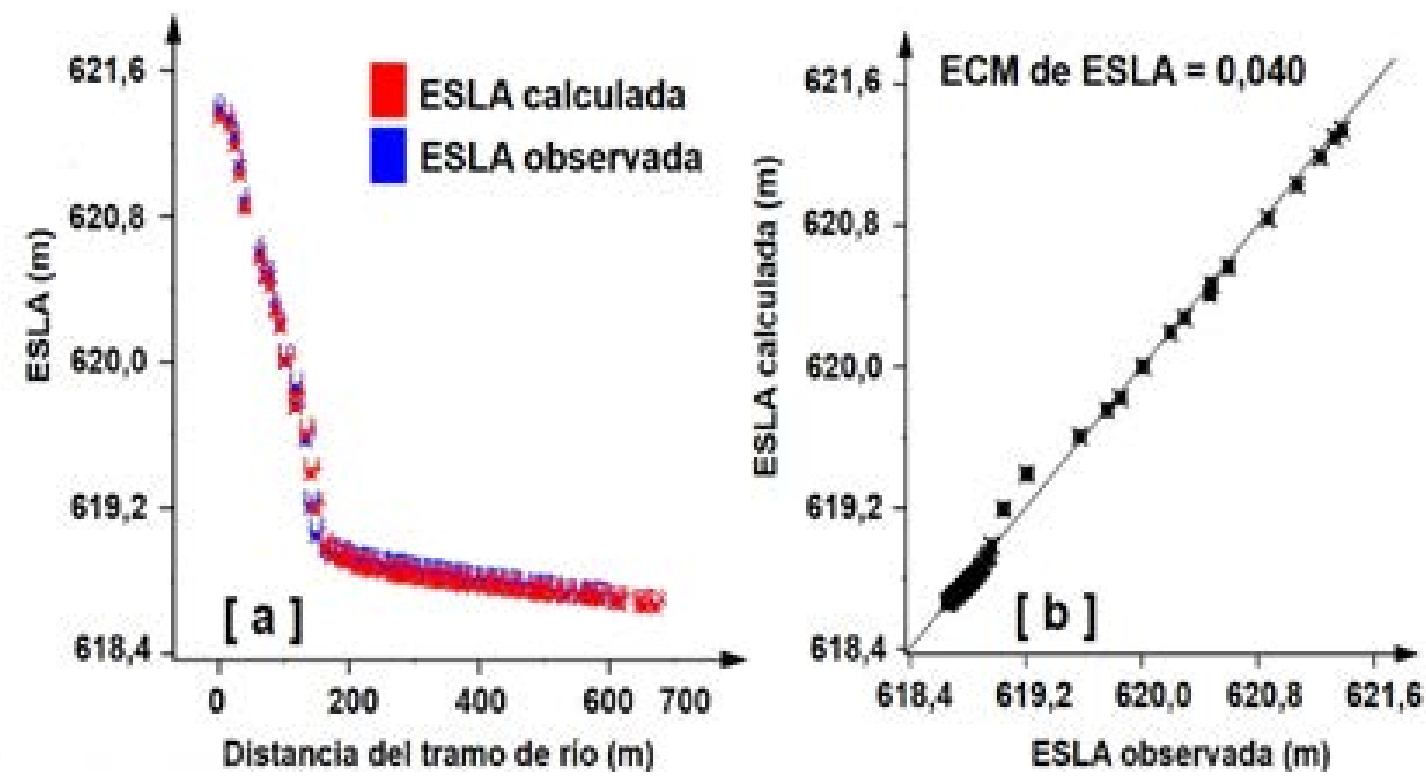

Fig. 3. a. asociación entre ESLA calculada por el software vs. observada en las campañas de campo, y b. ECM de ESLA entre el modelo calculado por el software y el observado en las campañas de campo

Fuente: elaboración propia. 
Tabla 1. Resultados de las simulaciones de caudal, el ECM de ESLA y el resultado de correlación entre ESLA calculada y observada

\begin{tabular}{|c|c|c|c|c|c|}
\hline $\begin{array}{l}\text { Caudal simulado } \\
\qquad\left(\mathrm{m}^{3} \mathrm{~s}^{-1}\right)\end{array}$ & $\begin{array}{l}\text { ECM de } \\
\text { ESLA (m) }\end{array}$ & $\mathbf{R}^{2}$ & Caudal simulado $\left(\mathrm{m}^{3} \mathrm{~s}^{-1}\right)$ & $\begin{array}{l}\text { ECM de } \\
\text { ESLA (m) }\end{array}$ & $\mathbf{R}^{2}$ \\
\hline 0,1 & 0,789 & 0,6201 & $\begin{array}{c}11,49 \text { caudal observado } \\
\text { aguas arriba }\end{array}$ & 0,044 & 0,9990 \\
\hline 0,2 & 0,768 & 0,6242 & $\begin{array}{c}11,50 \text { caudal observado } \\
\text { aguas abajo }\end{array}$ & 0,040 & 0,9992 \\
\hline 0,3 & 0,732 & 0,6306 & 12 & 0,088 & 0,9981 \\
\hline 0,4 & 0,721 & 0,6348 & 14 & 0,109 & 0,9989 \\
\hline 0,5 & 0,716 & 0,6511 & 16 & 0,135 & 0,9989 \\
\hline 0,8 & 0,712 & 0,6525 & 18 & 0,119 & 0,9922 \\
\hline 1 & 0,697 & 0,6540 & 20 & 0,141 & 0,9930 \\
\hline 3 & 0,557 & 0,8209 & 40 & 0,532 & 0,9611 \\
\hline 4 & 0,556 & 0,8489 & 60 & 0,731 & 0,9315 \\
\hline 6 & 0,138 & 0,9836 & 80 & 0,937 & 0,9073 \\
\hline 8 & 0,081 & 0,9936 & 100 & 1,210 & 0,9310 \\
\hline 10 & 0,283 & 0,9928 & 200 & 1,937 & 0,7648 \\
\hline
\end{tabular}

Fuente: elaboración propia.

Es importante mencionar que el buen ajuste encontrado entre los caudales calculados con los observados también exhibió un aumento del ECM de ESLA, conforme aumentan o disminuyen las simulaciones con respecto al caudal calibrado. Las simulaciones inferiores a $4\left(\mathrm{~m}^{3} \mathrm{~s}^{-1}\right)$ mostraron un ЕСм de ESLA por arriba de los 0,5 m, mientras que las simulaciones superiores a 100 y $200\left(\mathrm{~m}^{3} \mathrm{~s}^{-1}\right)$ presentaron un ECM de ESLA por arriba de $1,2 \mathrm{~m}$ (Tabla 1). Es necesario para futuras investigaciones diseñar campañas de campo para validar los caudales muy bajos y altos, dentro del modelado hidráulico.

\section{Simulación de hábitat físico}

La relación de caudal y hábitat presenta diferentes formas y valores absolutos de HPU entre las diferentes especies ( $N$. istlanum; $A$. cf. aeneus; P. butleri y I. furcidens, Figura 5). La cantidad de aptitud del hábitat disminuye con el aumento de los caudales. La distribución horizontal calculada mostró las mayores velocidades y profundidades en el centro del canal y tiende a disminuir hacia las orillas del río. Además, se encontró que en los caudales simulados el aumento HPU se produce cerca del banco derecho e izquierdo del río, y solo para la especie I. furcidens exhibió un aumento de HPU que se extiende en el tramo seleccionado, a caudales $>4\left(\mathrm{~m}^{3}\right.$ $\mathrm{s}^{-1}$ ) (Figura 4). La superficie de HPU para las especies de peces modelados fue sobre-predichos a flujos más bajos de $4\left(\mathrm{~m}^{3} \mathrm{~s}^{-1}\right)$.

La distribución del HPU obtenida de las simulaciones a lo largo del tramo de estudio mostró que en aguas arriba donde inicia el tramo de estudio con pendientes de $1,60 \%$ se tiene la condición de hábitat más pobre y el aumento de HPU para las especies se mueve hacia aguas abajo del tramo de estudio, donde se mejorará la condición de aptitud del hábitat con pendientes de 0,04 y $0,01 \%$ para las especies seleccionadas (Figura 4).

Un hallazgo importante en la simulación de hábitat físico para las cuatro especies fue la evidencia de distintas selecciones de hábitat en términos de profundidad, velocidad y tipo de sustratos. 


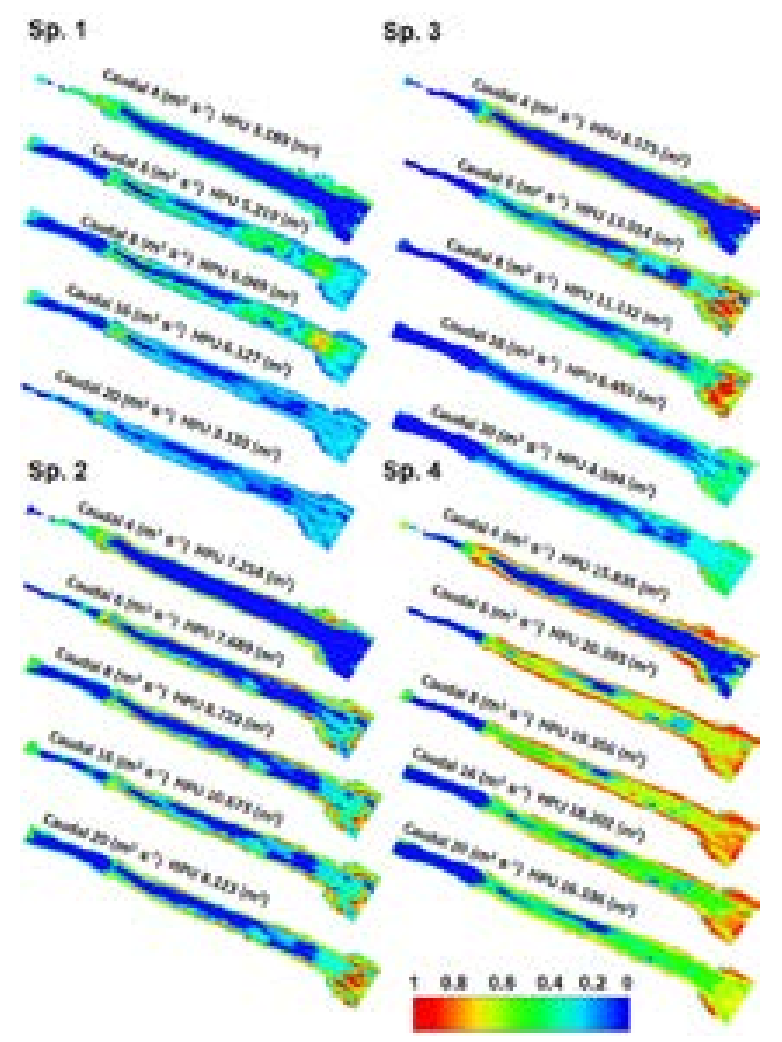

Fig. 4. Simulaciones de hábitat físico y la estimación del HPU

Fuente: elaboración propia.

Nota: el color rojo define el máximo de idoneidad (valor uno), y se degrada hasta alcanzar un color azul (valor cero). Sp. $1=N$. istlanum, Sp. $2=$ A. cf. aeneus, Sp. $3=$ P. butleri y Sp. $4=I$. furcidens

\section{Hábitat Potencial Útil vs. caudal simulado}

Las curvas (caudal/HPU) mostraron un gradiente más elevado de HPU para P. butleri y I. furcidens, pero tal diferencia fue menos notable para $N$. istlanum y A. cf. aeneus. Las curvas producidas presentaron, en general, una misma tendencia ( $\mathrm{Fi}$ gura 5). En los resultados se observó que los caudales simulados $<1\left(\mathrm{~m}^{3} \mathrm{~s}^{-1}\right)$ no producen suficiente representación de HPU; por su parte, las simulaciones entre 1 y $6\left(\mathrm{~m}^{3} \mathrm{~s}^{-1}\right)$ evidenciaron un crecimiento rápido de HPU para las cuatro especies, y las simulaciones de 6 a $16\left(\mathrm{~m}^{3} \mathrm{~s}^{-1}\right)$, las especies $N$. istlanum; A. cf. aeneus y I. furcidens, muestran un crecimiento lineal con relación a los caudales.

La especie $P$. butleri alcanzó su máximo HPU en los caudales simulados a 6 y $7\left(\mathrm{~m}^{3} \mathrm{~s}^{-1}\right)$ y I. furcidens a $6\left(\mathrm{~m}^{3} \mathrm{~s}^{-1}\right)$. Estas dos especies de la familia Poeciliidae presentaron similitudes de caudal a la especie
G. atripinnis, gracias a un estudio realizado en la cuenca baja del río Duero en México por [50]. Las especies $N$. istlanum alcanzaron su máximo HPU a $8\left(\mathrm{~m}^{3} \mathrm{~s}^{-1}\right)$ y A. cf. aeneus a $15\left(\mathrm{~m}^{3} \mathrm{~s}^{-1}\right)$ (Figura 5). Las variables hidráulicas modeladas (profundidad, velocidad y tipo de sustrato) cuando poseen un alto HPU para una determinada especie se debe al tamaño de la celda o pixel, resultado de las secciones transversales del levantamiento topográfico. Las cuatro especies estudiadas mostraron diferentes variaciones en HPU que dependen del caudal.

\section{Simulaciones del régimen hidráulico actual}

La serie de tiempo (1971-2001) entre el caudal mes/ HPU para las cuatro especies seleccionadas muestra que entre enero y mayo es el periodo de caudales decrecientes dentro del río, marcado por la estación seca. Las condiciones de aptitud de hábitat 
para las cuatro especies se analizan desde una evaluación general, considerando que, ordinariamente, durante el periodo seco en la primavera, el HPU es óptimo porque representa la condición de hábitat adecuada para la fecundación, el desarrollo embrionario, el nacimiento y el crecimiento de las especies [51] y [52].

Para las cuatro especies, estas fases las llevan a cabo entre marzo y mayo; por lo tanto, la primavera será un periodo de hábitat sensible para la etapa de la vida de estas especies [53]-[55] (Figura 6). Solo la especie $N$. istlanum presentó un HPU muy pobre para esta época, ocasionado por la retención de agua por las presas, lo que acerca los caudales del régimen hidrológico actual a un valor cero de caudal. Lo contrario ocurre en la época seca, en la época estival (lluvias), temporada cuando se presentan los mayores caudales de julio a octubre; en estos meses se muestra una disminución del HPU para las cuatro especies seleccionadas (Figura 6), hecho que confirma que un mayor caudal no representa un aumento de HPU. Estudios anteriores del río describen este comportamiento hidrológico de acuerdo con [26]. Es importante destacar que la toma de data para la construcción de las curvas de idoneidad de las especies seleccionadas fue conseguida, en gran parte del tiempo, durante la temporada de estiaje, cuando el río presenta menor caudal; esto ocasiona una incertidumbre al simular el HPU de los caudales altos.

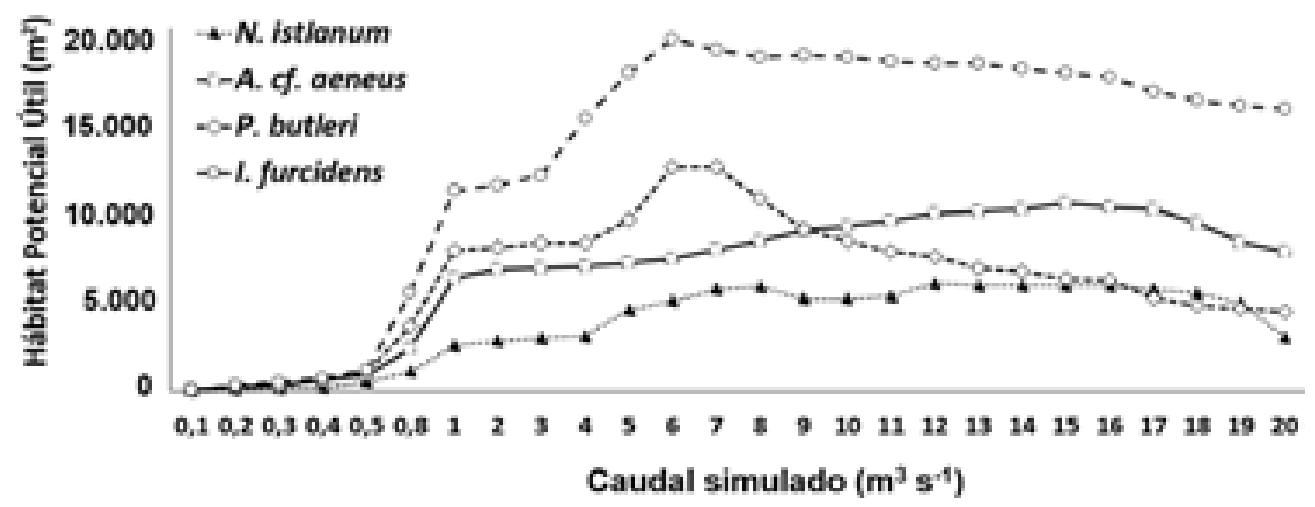

Fig. 5. Curvas HPU vs. caudales simulados por iRIC 1.5 para las especies.

Fuente: elaboración propia.

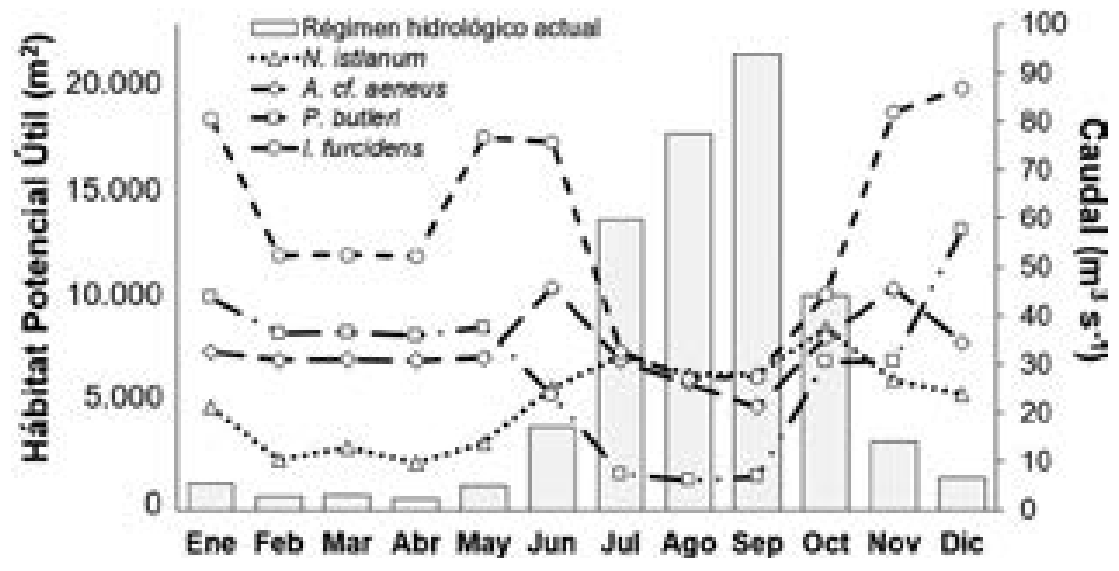

Fig. 6. Distribución promedio mensual del régimen hidrológico actual (1971-2001) y su relación con las simulaciones de caudal y su Hábitat Potencial Útil para las especies

Fuente: elaboración propia. 
Esta serie temporal de julio a octubre muestra el decaimiento de HPU e ilustra que habrá un estado de hábitat muy pobre para las cuatro especies [29]. Las relaciones entre las series temporales y el HPU son una parte fundamental para los estudios hidrobiológicos, y es necesario establecer las series temporales de HPU en condición de referencia de caudal natural en comparación con diferentes escenarios, como la regulación del caudal ocasionada por la retención de las presas, la extracción de agua y el cambio climático [26] y [56]. Los caudales simulados de las series temporales presentados en este estudio servirán para apoyar a los gestores en la toma de decisiones para administrar de mejor manera los recursos hídricos del río Ayuquila-Armería.

El sistema analítico iRIC 1.5 constituye una herramienta provechosa para el ordenamiento y manejo de los recursos hídricos. El modelado bidimensional es potencialmente capaz de simular patrones de flujos complejos de acuerdo con [18], [57] y [58] y proporciona información detallada con respecto al patrón de flujo, la velocidad y la tensión de arrastre del lecho del río. Pero también presentó una serie de sesgos en la simulación de hábitat, por ejemplo, una vez calibrado el software a un caudal circulante (obtenido de la campaña de campo), se intuye (como verdad) que con la misma calibración de este caudal circulante se pueden estimar los caudales circulantes (inferiores $1 \mathrm{~m}^{3} \mathrm{~s}^{-1}$ ) y los extremos (mayor $100 \mathrm{~m}^{3} \mathrm{~s}^{-1}$ ); esto ocasiona una serie de incertidumbre en los resultados de la simulación de hábitat. La toma de datos extremos está relacionada con aspectos de seguridad personal (mayor profundidad y velocidad en el río, que lo hace inaccesible), lo cual imposibilita la toma de datos.

Las simulaciones de hábitat físico en este estudio, de acuerdo con la IFIM, mostró una eficaz relación entre los parámetros físicos del hábitat en diferentes escalas y simulaciones de caudal. Esta relación funcional entre el caudal y el HPU permitió la integración del hábitat acuático, así como la evaluación del efecto potencial del régimen de caudal, utilizando series temporales [59]. Estos resultados dentro de la IFIM no son extrapolables, ya que se han utilizado como indicador del tramo seleccionado, de manera que no es válido para otros tramos o estudios de simulación de hábitat físico dentro del río. Es necesario llevar a cabo estudios de transferibilidad de los criterios de idoneidad, cuantificando el hábitat hidráulico y utilizando el método de [60], el cual puede emplearse para determinar la calidad y la cantidad de hábitat en otra corriente o tramos seleccionados.

En la actualidad, en México y en Latinoamérica la aplicación de este tipo de métodos no es común y no existen simulaciones de hábitat físico para las especies seleccionadas, que podrían ser utilizadas para establecer comparaciones.

\section{Conclusión}

Este es uno de los primeros estudios que identifica especies de peces neotropicales para su uso en estudios relacionados con la simulación de hábitat físico. Esto amplía el uso de modelos de predicción y la capacidad de predecir cuantitativamente los impactos biológicos de los cambios en las variables hidráulicas en ambientes acuáticos.

La simulación de hábitat físico aparece en México y en Latinoamérica como un campo nuevo de investigación que debe fomentarse, ya que proporciona soluciones razonadas para salvaguardar la destacada biodiversidad de sus ecosistemas acuáticos, que se encuentran en proceso acelerado de degradación. Esta propuesta de simulación de hábitat físico permitirá a los gestores de decisiones en el río Ayuquila-Armería -como lo indica la Norma Mexicana NMX-AA-159-SCFI- proteger y conservar los ecosistemas acuáticos desde un punto de vista más ecológico.

El software iRIC 1.5 en su plataforma FaSTMECH fue adecuado en este estudio para simular las características del hábitat físico en dos dimensiones, lo que permitió cambiar el tamaño de la malla y variar la parametrización del modelo, y así mejorar los resultados de la simulación dentro del río Ayuquila-Armería y presentar un ajuste a las variables hidráulicas en el proceso de calibración. Las elevaciones de la lámina de agua observada de las 62 secciones transversales, que se obtuvieron del levantamiento topográfico y la combinación de las curvas de idoneidad propuestas, permitieron con mayor detalle describir las simulaciones del hábitat físico dentro del tramo seleccionado. 
Un aporte importante en este estudio fueron las curvas de idoneidad de las especies $N$. istlanum; $A$. cf. aeneus; $P$. butleri y I. furcidens; estas no limitarán el uso de modelos de predicción y la capacidad de predecir de manera cuantitativa los impactos biológicos por la carencia de información, y sí servirán para futuras simulaciones de hábitat físico a lo largo de las vertientes (Atlántico y Pacifico). N. istlanum se localiza en el occidente mexicano, $A$. cf. aeneus utiliza ambas vertientes de las Américas y las Indias Occidentales; desde Veracruz en México hasta Costa Rica y Colombia. El P. butleri utiliza la vertiente del Pacífico en México y Mesoamérica, desde el río Fuerte, en Sonora, hasta la boca del río Comasagua, en El Salvador. I. furcidens se localiza en la vertiente del Pacífico, en los ríos Ameca, Armería, Marabasco y Coahuayana.

Para finalizar, estudios como este exponen las ventajas de seleccionar la técnica de simulación de hábitat físico, que se establece en la metodología de la Norma Mexicana NMX-AA-159-SCFI-2012 en su apéndice normativo E, para salvaguardar los sistemas de ríos en México.

\section{Agradecimentos}

Un agradecimiento al proyecto Manejo y conservación de los recursos naturales del río Ayuquila-Armería del Departamento de Ecología y Recursos Naturales de la Universidad de Guadalajara, por su acceso a la información. Se agradece a las aportaciones críticas del referee encargado de la evaluación de este artículo.

\section{Referencias}

[1] D. J. Booker y M. J. Dunbar, "Application of physical habitat simulation (PHABSIM) modelling to modified urban river channels," River Research Applications, vol. 20, no. 2, pp. 167-183, 2004. DOI: https://doi. org/10.1002/rra.742.

[2] M. C. Freeman, Z. H. Bowen, K. D. Bovee y E. R. Irwin, "Flow and habitat effects on juvenile fish abundance in natural and altered flow regimes," Ecological Applications, vol. 11, no. 1, pp. 179-190, 2001. DOI: https://doi. org/10.2307/3061065

[3] R. E. Tharme, "A global perspective on environmental flow assessment: emerging trends in the development and application of environmental flow methodologies for rivers," River Research and Applications, vol. 19, pp. 397-441, 2003. DOI: https://doi.org/10.1002/rra.736

[4] M. C. Acreman y M. J. Dunbar, "Defining environmental river flow requirements? A review," Hydrology Earth System Sciences, vol. 8, pp. 861-876, 2004. DOI: https://doi.org/10.5194/hess-8-861-2004

[5] A. H. Arthington, S. E. Bunn, N. L. Poff y R. J. Naiman, "The challenge of providing environmental flow rules to sustain river ecosystems," Ecological Applications, vol. 16, no. 4, pp. 1311-1318, 2006. DOI: https:// doi.org/10.1890/1051-0761(2006)016[1311:TCOPE$\mathrm{F}] 2.0 . \mathrm{CO} ; 2$

[6] I. Maddock, "The importance of physical habitat assessment for evaluating river health," Freshwater Biology, vol. 41, pp. 373-391, 1999. DOI: https://doi.org/10.1046/j.1365-2427.1999.00437.x

[7] T. Oberdorff, D. Pont, B. Hugueny y J.-P. Porcher, "Development and validation of a fish-based index for the assessment of "river health" in France," Freshwater Biolo$g y$, vol. 47, no. 9, pp. 1720-1734, 2002. DOI: https://doi. org/10.1046/j.1365-2427.2002.00884.x

[8] A. Mouton, H. Meixner, P. L. Goethals, N. De Pauw y H. Mader, "Concept and application of the usable volume for modelling the physical habitat of riverine organisms," River Research Applications, vol. 23, no. 5, pp. 545-558, 2007. DOI: https://doi.org/10.1002/rra.998

[9] C. A. Frissell, W. J. Liss, C. E. Warren y M. D. Hurley, "A hierarchical framework for stream habitatclassification: Viewing streams in a watershed context," Environmental Management, vol. 10, no. 2, pp. 199-214, 1986. DOI: https://doi.org/10.1007/BF01867358

[10] P. W. Downs y G. M. Kondolf, "Post-project appraisals in adaptive management of river channel restoration," Environmental Management, vol. 29, no. 4, pp. 477-496, 2002. DOI: https://doi.org/10.1007/s00267-001-0035-X

[11] D. Im., H. Kang, K. H. Kim y S. U. Choi, "Changes of river morphology and physical fish habitat following weir removal," Ecological Engineering, vol. 37, no. 6, pp, 883-892, 2011. DOI: https://doi.org/10.1016/j.ecoleng.2011.01.005

[12] J. M. Nestler, R. A. Goodwin, D. L. Smith y J. J. Anderson, "A Mathematical and Conceptual Framework for Ecohydraulics," en Hydroecology and Ecohydrology: Past, Present and Future, P.J. Wood., D.M. Hannah y J.P. Sadler, Eds. Chichester, Reino Unido: John Wiley \& Sons, 2008, pp. 205-224. DOI: https://doi. org/10.1002/9780470010198.ch12

[13] J. M. Diez Hernández y L. Burbano Burbano, "Revisión de los modelos eco-hidráulicos uni-dimensionales (1D) y bidimensionales (2d) en corrientes fluviales," Avances en Recursos Hidráulicos, no. 15, pp. 75-88, 2007. 
[14] S. Rice, S. Little, P. Wood, H. Moir y D. Vericat, “The relative contributions of ecology and hydraulics to ecohydraulics," River Research and Applications, vol. 26, no. 4, pp. 363-366, 2010. DOI: https://doi.org/10.1002/ rra.1369

[15] J. M. Nestler, M. J. Stewardson, D. J. Gilvear, A.J. Webb y D. L. Smith, "Ecohydraulics exemplifies the emerging "paradigm of the interdisciplines"," Journal of Ecohydraulics, vol. 1, no. 1-2, pp. 5-15, 2016. DOI: https://doi.org/10.1080/24705357.2016.1229142

[16] I. Maddock, A. Harby, P. Kemp y P. Wood, "Ecohydraulics: an introduction," en Ecohydraulics: An integrated approach, I. Maddock., A. Harby., P. Kemp y P. Wood, Eds. Chichester, Reino Unido: John Wiley \& Sons, 2013, pp. 1-6. DOI: https://doi.org/10.1002/9781118526576.ch1

[17] R. T. Milhous, D. L. Wegner y T. Waddle. User's guide to the physical habitat simulation system, Washington, D. C., Estados Unidos: U. S. Fish and Wildlife Service, 1981.

[18] M. Gard, "Comparison of spawning habitat predictions of PHABSIM and River2D models," International Journal of River Basin Management, vol. 7, no. 1, pp. 55-71, 2009. DOI: https://doi.org/10.1080/1571512 4.2009.9635370

[19] J. H. Lee, J. T. Kil y S. Jeong, "Evaluation of physical fish habitat quality enhancement designs in urban streams using a 2D hydrodynamic model," Ecological Engineering, vol. 36, no. 10, pp. 1251-1259, 2010. DOI: https://doi.org/10.1016/j.ecoleng.2010.05.004

[20] D. Ayllón, A. Almodóvar, G. G. Nicola, I. Parra y B. Elvira, "A new biological indicator to assess the ecological status of Mediterranean trout type streams," Ecological Indicators, vol. 20, pp. 295-303, 2012. DOI: https://doi.org/10.1016/j.ecolind.2012.02.028

[21] P. Steffler y J. Blackburn. Two-dimensional depth averaged model of river hydrodynamics and fish habitat, Introduction to Depth Averaged Modeling and User's Manual, Alberta, Canadá: University of Alberta, 2002.

[22] I. Dongkyun, K. Hyeongsik, K. Kyu-Ho y C. Sung-Uk, "Changes of river morphology and physical fish habitat following weir removal," Ecological Engineering, vol. 37, no. 6, 883-892, 2011. DOI: https://doi.org/10.1016/j. ecoleng.2011.01.005

[23] I. Boavida, J. M. Santos, C. Katopodis, M. T. Ferreira y A. Pinheiro, "Uncertainty in predicting the fishresponse to two-dimensional habitat modeling using field-data," River Research and Applications, vol. 29, pp. 1164-1174, 2013. DOI: https://doi.org/10.1002/rra.2603
[24] P. Parasiewicz et al., "Applications of the MesoHABSIM Simulation Model," en Ecohydraulics An Integrated Approach, I. Maddock., A. Harby., P. Kemp y P. Wood, Eds. Chichester, Reino Unido: JohnWiley \& Sons, 2013, pp. 109124. DOI: https://doi.org/10.1002/9781118526576.ch6

[25] J. G. Holmquist y T. J. Waddle, "Predicted macroinvertebrate response to water diversion from a montane stream using two-dimensional hydrodynamic models and zero flow approximation," Ecological Indicators, vol. 28, 115-124, 2013. DOI: https://doi.org/10.1016/j. ecolind.2012.03.005

[26] K. D. Bovee et al., "Stream habitat analysis using the instream flow incremental methodology, Fort Collins CO, U.S. Geological Survey," Biological Resources Division Information and Technology Report USGS/BRD, VIII, 1998.

[27] I. E. Oliveira, D. D. Da Silva, H. A. S. Guedes, J. A. Dergam y C.B.D. Ribeiro, "One- and two-dimensional ecohydraulic modeling of formoso river (MG)," Engenharia Agrícola, vol. 36, no. 6, 1050-1062, 2016. DOI: https://dx.doi.org/10.1590/1809-4430-eng.agric. v36n6p1050-1062/2016

[28] DOF, "Norma Mexicana NMX-AA-159-SCFI. Que establece el procedimiento para la determinación del caudal ecológico en cuencas hidrológicas," Diario Oficial de la Federación, 2012.

[29] D. Meza-Rodríguez et al., "Propuesta de caudal ecológico en la cuenca del Río Ayuquila-Armería en el Occidente de México," Latin American Journal of Aquatic Research, vol. 45, no. 5, 1017-1030, 2017. DOI: https://dx.doi.org/10.3856/vol45-issue5-fulltext-17

[30] R.L.M. Martínez, A. Carranza y G. Micaela, “Aquatic ecosystem pollution of the Ayuquila River, Sierra de Manantlán Biosphere Reserve, México”, en Aquatic Ecosystems of Mexico: Status and Scope, M. Munawar., S.G. Lawrence., I.F. Munawar y D.F. Malley, Eds. Leiden, Países Bajos: Ecovision World Monograph Series, 2000, pp. 165-181.

[31] J. Lyons y S. Navarro-Pérez, "Fishes of the Sierra de Manantlán, West-Central Mexico," The Southwestern Naturalist, vol. 35, no. 1, pp. 32-46, 1990. DOI: https:// doi.org/10.2307/3671983

[32] E. Santana et al., "Contaminación, aprovechamiento y conservación de los recursos acuáticos del Río Ayuquila, Reserva de la Biosfera Sierra de Manantlán, Jalisco-Colima," Tiempos de Ciencia, no. 30, pp. 29-38, 1993.

[33] D. Meza-Rodríguez et al., "Régimen natural de caudales del río Ayuquila-Armería en el occidente de México," Terra Latinoamericana, vol. 35, no. 3, pp. 203-217, 2017. DOI: https://dx.doi.org/10.28940/terra.v35i3.224 
[34] M.G. Wolman, "A method of sampling coarse river-bed material," Transactions American Geophysical Union, vol. 35, no. 6, pp. 951-956, 1954. DOI: https://doi. org/10.1029/TR035i006p00951

[35] V. T. Chow. Open-Channel Hydraulics, Nueva York, Estados Unidos: MacGraw-Hill, 1959.

[36] R. R. McDonald, J. M. Nelson y J. P. Bennett, Multi-dimensional surface-water modeling system user's guide, U.S. Geological Survey Techniques and Methods, 6-B2, 2005.

[37] J. Chang-Lae y Y. Shimizu, "Numerical simulation of relatively wide, shallow channels with erodible banks," Journal of Hydraulic Engineering, vol. 131, no. 7, pp. 565-575, 2005. DOI: https://doi.org/10.1061/ (ASCE)0733-9429(2005)131:7(565)

[38] J.M. Nelson et al., "The international river interface cooperative: Public domain flow and morphodynamics software for education and applications," Advances in Water Resources, vol. 93, pp. 62-74, 2016. DOI: https:// doi.org/10.1016/j.advwatres.2015.09.017

[39] Stillwater Sciences, Lower Tuolumne River Instream Flow Studies: Pulse Flow Study Report. Berkeley, Estados Unidos: Turlock Irrigation District, Modesto Irrigation District, 2012.

[40] T. A. Kenney y M. L. Freeman, "Two-dimensional streamflow simulations of the Jordan River, Midvale and West Jordan, Utah," en Prepared in cooperation with the U.S. Environmental Protection Agency. Scientific Investigations Report 2011-5043, 2011. DOI: https:// doi.org/10.3133/sir20115043

[41] R. C. Hilldale, D. M. Mooney y K. L. Collins, Identifying Stream Habitat Features With a Two-Dimensional Hydraulic Model. Yakima River Basin Water Storage Feasibility Study, Washington," Reclamation. Managing Water in the West, Technical Series, no. TS-YSS-12.2007.

[42] G. B. Pastemack y A. Senter, "21st Century instream flow assessment framework for mountain streams," 2011. [En línea]. Disponible en: https://ww2.energy. ca.gov/2013publications/CEC-500-2013-059/CEC-5002013-059.pdf

[43] C. F. Martínez-Capel y D. L. García de Jalón, “Desarrollo de curvas de preferencia de microhábitat para Leuciscus pyrenaicus y Barbus bocagei por buceo en el río Jarama (Cuenca del Tajo)," Limnetica, vol. 17, no. 1, pp. 71-83, 1999.

[44] H. J. Moir, C. N. Gibbins, C. Soulsby y A. F. Youngson, "PHABSIM modelling of Atlantic salmon spawning habitat in an upland stream: testing the influence of habitat suitability indices on model output," River Research and Applications, vol. 21, no. 9, pp. 1021-1034, 2005. DOI: https://doi.org/10.1002/rra.869
[45] D. Tonina, J. A. McKean, C. Tang y P. Goodwin, "New tools for aquatic habitat modeling," en Balance and uncertainty - Water in a changing world: Proceedings of the 34th IAHR World Congress, 33rd Hydrology and Water Resources Symposium, and 10th Conference on Hydraulics in Water Engineering, E.M. Valentine, Ed. Brisbane, Australia. Engineers Australia, 2011, pp. 3137-3144.

[46] R. Benjankar, D. Tonina y J. McKean, "One-dimensional and two-dimensional hydrodynamic modeling derived flow properties: impacts on aquatic habitat quality predictions," Earth Surface Processes and Landforms, vol. 40, no. 3 pp. $340-356,2015$. DOI: https://doi. org/10.1002/esp.3637

[47] K. D. Bovee, “The Incremental Method of Assessing Habitat Potential for Cool Water Species, with Management Implications," American Fisheries Society Special Publication, vol. 11, pp. 340-346, 1978.

[48] S. Y. Jaw y C. J. Chen, "Present status of second-order closure turbulence models. I: overview," Journal of Engineering Mechanics, vol. 124, no. 5, pp. 485-501, 1998. DOI: https://doi.org/10.1061/(ASCE)0733-9399 (1998)124:5(485)

[49] Comisión Nacional del Agua (Conagua), Secretaría del Medio Ambiente y Recursos Naturales e Instituto Mexicano de Tecnología del Agua. Banco Nacional de Datos de Aguas Superficiales (BANDAS). Jiutepec, México, 2008. [En línea]. Disponible en: http://www. conagua.gob.mx/CONAGUA07/Contenido/Documentos/Portada\%20BANDAS.htm

[50] F. Armas-Vargas et al., "Proposing environmental flow based on physical habitat simulation for river fish species in the Lower Duero River Basin, Mexico," Hidrobiológica, vol. 27, no. 2, pp. 185-200, 2017. DOI: https://doi. org/10.24275/uam/izt/dcbs/hidro/2017v27n2/Armas

[51] M. R. Rush. Peces dulceacuícolas de México. Ciudad de México: Editorial Comisión Nacional para el Conocimiento y Uso de la Biodiversidad, 2009.

[52] G. Ruiz-Campos, “Catálogo de peces dulceacuícolas de Baja California Sur, Secretaria de Medio Ambiente y Recursos Naturales,” Baja California, México: Instituto Nacional de Ecología, 2012.

[53] J. Lyons y N. Mercado-Silva, "Patrones taxonómicos y ecológicos entre comunidades de peces en ríos y arroyos en el Oeste de Jalisco, México," Anales del Instituto de Biología. Serie Zoología, vol. 70, no. 2, pp. 169-190, 1999.

[54] N. Mercado-Silva, J. D. Lyons, G. M. Salgado y M. N. Medina, "Validation of a fish-based index of biotic integrity for streams and rivers of central Mexico,, Reviews in Fish Biology and Fisheries, vol. 12, no. 2-3, pp. 179-191, 2002. DOI: https://doi.org/10.1023/A:1025099711746 
[55] J. Luna-Figueroa, F. Díaz y S. Espina, "Preferred temperature of the mexican native cichlid Cichlasoma istlanum (Jordan y Snyder, 1899)," Hidrobiológica, vol. 14, no. 4, pp. 271-275, 2003.

[56] R. T. Milhous, J. M. Bartholow, M. A. Updike y A. R. Moos. Reference Manual for Generation and Analysis of Habitat Time Series-Version II. Washington, D. C., Estados Unidos: Fish and Wildlife Service, 1990. [En línea]. Disponible en: https://apps.dtic.mil/dtic/tr/fulltext/u2/a322929.pdf

[57] I. G. Jowett y M. J. Duncan, "Effectiveness of 1D and 2D hydraulic models for instream habitat analysis in a braided river," Ecological Engineering, vol. 48, pp. 92-100, 2012. DOI: https://doi.org/10.1016/j.ecoleng.2011.06.036
[58] D. Tonina y K. Jorde, "Chapter 3. Hydraulic Modelling Approaches for Ecohydraulic Studies: 3D, 2D, 1D and Non-Numerical Models," en Ecohydraulics, I. Maddock., A. Harby., P. Kemp y P. Wood, Eds. Chichester, Reino Unido: John Wiley \& Sons, 2013, pp. 31-74. DOI: https://doi.org/10.1002/9781118526576.ch3

[59] T. R. Payne, "A roadmap for PIER research on instream flow determinations for hydropower applications in California,” 2004. [En línea]. Disponible en: https://ucciee.org/downloads/HydropowerRoadmap.Payne.pdf

[60] J. A. Thomas y K. D. Bovee, "Application and testing of a procedure to evaluate transferability of habitat suitability criteria," Regulated Rivers: Research and Management, vol. 8, no. 3, pp. 285-294, 1993. DOI: https://doi. org/10.1002/rrr.3450080307 